\title{
What We are Learning from Real World Data on Rivaroxaban for the Treatment of VTE: An Overview
}

Pierpaolo Di Micco ${ }^{1 *}$, Francesco Dentali², Nicola Mumoli ${ }^{3}$, Matteo Giorgi Pierfranceschi ${ }^{4}, \mathrm{Maria} \mathrm{Amitrano}^{5}$, Fulvio Pomero ${ }^{6}$, Giorgio Vescovo $^{7}$, Gualberto Gussoni ${ }^{8}$, Daniela Mastroiacovo ${ }^{9}$ and Andrea Fontanella ${ }^{1}$

${ }^{1}$ UOC Medicine, Fatebenefratelli Hospital of Naples, Italy

${ }^{2}$ Department of Medicine and Surgery, University of Insubria, Varese, Italy

${ }^{3}$ UO Internal Medicine, Hospital of Portoferraio, Livorno, Italy

${ }^{4}$ Emergency Department, Hospital of Val d'Arda, Piacenza, Italy

${ }^{5}$ UOS Angiology, AO Moscati, Avellino, Italy

${ }^{6}$ Department of Internal Medicine, Santa Croce and Carle Hospital, Cuneo, Italy

${ }^{7}$ UO Medicine, Sant'Antonio Hospital, Padua, Italy

${ }^{8}$ Centro Sudi FADOI, Milan, Italy

`UO Angiology, PO Avezzano, Aquila, Italy

\begin{abstract}
Rivaroxaban is one of most common used DOACs in the treatment of VTE. Rivaroxaban has been tested in a series of premarketing studies and after it has been used in non-interventional studies and in real life studies.

All studies experienced that rivaroxaban shows relevant safety concerning main outcomes of VTE therapy as recurrent VTE and major bleedings, in particular in patients that show similar clinical characteristics to those selected for EINSTEIN studies. Suggested doses of rivaroxaban seem to be safe also in real life studies while non-conventional doses may be associated to increased rate of clinical complications as recurrent VTE and major bleedings. Rivaroxaban shows also good safety when used in real life studies in patients with clinical characteristics that differ from those of patients in the EINSTEIN studies.
\end{abstract}

\section{Background}

Venous thromboembolism (VTE) is a life-threatening disease that usually may appear as deep vein thrombosis (DVT) andlor pulmonary embolism (PE) [1]. DVT may appear also in other sites as upper limb or in abdominal vein but these conditions are rarer than lower limb DVT.

International guidelines of several scientific societies as American College of Chest Physician (ACCP) suggest to treat VTE with direct oral anticoagulants (DOACs) versus other anticoagulants as low molecular weight heparins, fondaparinux or antivitamin K (AVK) drugs both in acute phase and also for long term treatment [2]; other treatments as thrombolysis are reserved to PE with haemodinamyc instability with trend to cardiogenic shock [2]. Rivaroxaban is a DOACs that exerts its anticoagulant activity toward clotting factor Xa [3].

Its ability to treat VTE has been tested in several PHASE III studies as dose findings studies and as phase III clinical trials; moreover, the usefulness of rivaroxaban in VTE has been also tested in post marketing studies as non-interventional studies and as clinical reports and clinical series from real life. Based on this great documented experience in VTE-treatment in premarketing phase and in post marketing phase, actually rivaroxaban seems to be one of the most used anticoagulant drugs in the world in particular in the treatment of VTE. Here we report emerging useful clinical data for the daily clinical use of rivaroxaban in the treatment of VTE, starting from dose findings studies till real world studies.

\section{PHASE II and III Studies}

ODIXA DVT is the major dose finding study to test the ability of rivaroxaban to treat VTE [4]. In this study several doses of rivaroxaban have been tested in the treatment of acute phase of DVT (i.e. $10 \mathrm{mg}$ twice daily, $20 \mathrm{mg}$ twice daily, $30 \mathrm{mg}$ twice daily and $40 \mathrm{mg}$ daily respectively). The analysis of pooled data suggested that $20 \mathrm{mg}$ daily and $30 \mathrm{mg}$ daily were dosages associated to best outcomes as prevention of recurrent VTE and fatal PE and as major bleeding and fatal bleeding [4] .

EINSTEIN studies are a group of phase III studies in which rivaroxaban has been tested versus standard treatments of VTE (i.e. enoxaparin at therapeutic regimen and AVK drugs for the first days followed by AVK drugs alone when INR range was 2.0-3.0). All EINSTEIN studies were randomized trials on VTE.

EINSTEIN DVT enrolled only patients affected by DVT confirmed by objective methods and selected to the treatment with standard therapy (i.e. enoxaparin at therapeutic regimen and AVK drugs for the first days followed by AVK drugs alone when INR range was 2.03.0) vs. patients treated with rivaroxaban with single drug approach (i.e. $15 \mathrm{mg}$ twice daily for first 21 days after diagnosis, followed by 20 mg daily for long term therapy at 3-6-12 months) [5]. Results of this study underlined that rivaroxaban with single drug approach showed a non-inferiority vs. standard therapy regarding main outcomes (i.e. prevention of recurrent DVT, prevention of recurrences of any type of VTE, prevention of fatal PE, prevention of major bleeding and prevention of fatal bleeding). In a similar way the EINSTEIN PE study was designed to test the efficacy of rivaroxaban with single drug approach versus standard therapy of VTE in PE diagnosed

${ }^{*}$ Corresponding author: Pierpaolo Di Micco, UOC Medicina, Fatebenefratell Hospital of Naples, Italy, Tel: 393398 078146; E-mail: pdimicco@libero.it

Received November 09, 2017; Accepted November 13, 2017; Published November 20, 2017

Citation: Di Micco P, Dentali F, Mumoli N, Pierfranceschi MG, Amitrano M et al. (2017) What We are Learning from Real World Data on Rivaroxaban for the Treatment of VTE: An Overview J Blood Lymph 8: 192 doi:10.4172/2165 7831.1000192

Copyright: @ 2017 Di Micco P, et al. This is an open-access article distributed under the terms of the Creative Commons Attribution License, which permits unrestricted use, distribution, and reproduction in any medium, provided the original author and source are credited. 
with objective methods with or without associated DVT [6]. Also EINSTEIN PE study was a randomized study. This study testified that rivaroxaban was safe too and non-inferior to standard treatment for patients affected by PE. All main outcomes (i.e. prevention of recurrent DVT, prevention of recurrences of any type of VTE, prevention of fatal $\mathrm{PE}$, prevention of major bleeding, and prevention of fatal bleeding) rates were similar to standard therapy and there was a reduction of major bleedings. All patients recruited in the EINSTEIN PE study were affected by PE without haemodynamic instability [6]. The benefit and the safety of therapy of VTE with rivaroxaban in EINSTEIN studies were also analyzed in a pooled analysis [7].

Moreover, for the extended therapy of VTE, rivaroxaban was also tested in another trial, EINSTEIN extension $[8,9]$. EINSTEIN extension tested the safety of long term treatment with rivaroxaban in patients for whom there was equipoise with respect to the need for continued anticoagulation after the first $3 \backslash 6 \backslash 12$ months of treatment. Also, EINSTEIN extension was a randomized trial in which rivaroxaban 20 mg daily was tested vs. placebo in long term prevention of recurrent VTE $[8,9]$. However also this study testified that rivaroxaban was safe for the long term use in all main outcomes (i.e. prevention of recurrent DVT, prevention of recurrences of any type of VTE, prevention of fatal $\mathrm{PE}$, prevention of major bleeding, prevention of fatal bleeding) versus placebo $[8,9]$.

\section{Phase IV studies}

Non interventional studies: Xalia is the first non-interventional and observational study that tested the safety of rivaroxaban in the treatment of VTE [10]. In this study patients were selected for VTE therapy with rivaroxaban and its single drug approach vs. standard therapy (i.e. enoxaparin at therapeutic regimen and AVK for the first days followed by AVK alone when INR range was 2.0-3.0). In this study the clinical characteristics of patients selected for each treatment of VTE were at complete discretion of each physician. Results of this study showed that patients treated with rivaroxaban had $0.7 \%$ of major bleedings and $1.4 \%$ of VTE recurrences. These results are consistent with the results of EINSTEIN studies and confirm rivaroxaban safety in the treatment of VTE [10].

Moreover, another non-interventional, observational study on safety of rivaroxaban in VTE is ongoing in oncological patients and it is named COSIMO. Clinical data are actually not available because this study is currently recruiting patients.

\section{Registries}

Several information is available on the daily use of rivaroxaban in VTE treatment. This info flow derives from clinical experience of each physician and it is consultable for the scientific community in the Literature as clinical case reports or case series or clinical registries.

The most common registries that provide clinical information on VTE in the Literature are the DRESDEN NOAC registry, the SWIVTER registry and the RIETE registry.

The DRESDEN NOAC registry is a prospective regional registry in which patients in treatment with DOACs undergo prospective follow up. In a recent publication the data of 407 patients treated with rivaroxaban for VTE were analyzed [11]. Patients that performed single drug approach with rivaroxaban were nearly $93 \%$. Concerning main outcomes, a $1 \%$ rate of recurrent VTE was found and a $3.2 \%$ rate of major bleeding in the same group of patients was recorded. It is noteworthy that, after rivaroxaban discontinuation, major bleeding rates remained at a clinically relevant level (1\%/year), probably due to co-morbidities in this group of patients. Overall these data confirmed a good safety of the treatment with rivaroxaban in real life [11].

The SWIVTER registry investigated the three-months clinical outcomes in patients treated for VTE with rivaroxaban vs. standard therapy. Data available in this registry for 417 patients were analyzed and compared to data of 1645 patients treated with conventional therapy for VTE with propensity score analysis [12]. Results testified that the rate of major bleeding was similar in both groups $(0.5 \%$ in both groups) while a light improvement in prevention of recurrent VTE was available in the group treated with rivaroxaban compared to conventional therapy (1.2\% vs. $2-1 \%$ respectively) [12].

The RIETE registry enrolled patients affected by VTE. Clinical characteristics and outcomes of more than 70000 patients affected by VTE with objective methods were recorded. Several data are available in this registry for patients treated with rivaroxaban.

In a recent article published by Trujillo Santos et al. data collected in the RIETE registry in 1635 patients treated with DOACs for VTE were analyzed [13]; 1591 patients took rivaroxaban for treatment of VTE. Interesting data was given by the registry concerning rivaroxaban, in particular the article underlines that only $81 \%$ received rivaroxaban at suggested doses for therapy. In the group of patients with suggested doses the main outcomes as recurrent VTE or major bleeding showed a reduced rate than expected [13]. Different data appeared for patients treated with unconventional doses [13]. This subgroup of patients received different doses from $15 \mathrm{mg}$ BID in acute phase and $20 \mathrm{mg}$ OD for long term therapy of VTE and showed a higher rate of recurrent VTE and major bleeding [13]. It should be noted that unconventional dosages of any drug, and not only of rivaroxaban, are associated with higher risks.

Moreover also information on fragile patients treated with rivaroxaban is available in the RIETE registry. Fragile patients were defined as those with renal impairment with $\mathrm{ClCr}<50 \mathrm{ml} \backslash \mathrm{min}$, body weight $<50 \mathrm{~kg}$, age $>75$ years. In fragile patients rivaroxaban was choosen as main drug to treat VTE in nearly 350 cases in acute phase and in nearly 1000 patients for long term therapy [14]. In fragile patients, due to the complexity of the clinical conditions associated to VTE, there seemed to be a higher trend of bleeding and VTE recurrence rates compared to non-fragile patients. Yet, in this study, in fragile patients taking rivaroxaban there was a light reduction in VTE recurrences and in major bleedings compared to fragile patients taking other anticoagulant drugs [14].

In another article published by the RIETE investigators, data from patients treated for VTE with rivaroxaban that showed exclusion criteria for DOACs (i.e. ageing, renal impairment, metastatic cancer and so on) were analyzed. These patients seem to have a stronger trend to have clinical complications if compared to those that show classic clinical characteristic to receive DOACs [15]. Yet, although this trend is typical for all kind of anticoagulant drugs, a subgroup analysis for each anticoagulant drug was performed and showed that unconventional patients treated with rivaroxaban have a light reduction in VTE recurrenceand in major bleeding too [15].

\section{Conclusion}

Premarketing studies focused on the antithrombotic treatment of VTE with rivaroxaban found a good impact concerning main outcomes as recurrent VTE and major bleeding. These data have been confirmed by following studies also real life studies and non-interventional studies 
Citation: Di Micco P, Dentali F, Mumoli N, Pierfranceschi MG, Amitrano M, et al. (2017) What We are Learning from Real World Data on Rivaroxaban for the Treatment of VTE: An Overview J Blood Lymph 8: 192. doi:10.4172/2165-7831.1000192

and rivaroxaban has been confirmed as one of the main suggested drug to treat VTE also in international guidelines.

EINSTEIN studies does not show exclusion criteria to enroll patients affected by VTE. However, clinical characteristics of enrolled patients in EINSTEIN studies were similar to young patients affected by VTE in real life (i.e. age $<65$ year, normal kidney function, body weight $>50 \mathrm{~kg}$, no-cancer). The most of post-marketing studies, noninterventional-observational studies and clinical registries, enrolled patients with similar characteristics to those enrolled in EINSTEIN studies and results underlined a further reduction of clinical complications as recurrent VTE and major bleeding.

Yet, rivaroxaban showed a little decrease of its safety in main clinical outcomes if used with dosages other than those suggested by phase III clinical trials. Its ability in the treatment of patients affected by VTE at high risk complications as major bleeding and overall death should be better investigated by future studies.

\section{References}

1. Scheres LJJ, Brekelmans MPA, Beenen LFM, Büller HR, Cannegieter SC, et al. (2017) Sex-specific differences in the presenting location of a first venous thromboembolism. J Thromb Haemost 15: 1344-1350.

2. Kearon C, Akl EA, Ornelas J, Blaivas A, Jimenez D, et al. (2016) Antithrombotic Therapy for VTE Disease: CHEST Guideline and Expert Panel Report. Chest 149: 315-352.

3. Escolar G, Carne X, Arellano-Rodrigo E (2015) Dosing of rivaroxaban by indication: getting the right dose for the patient. Expert Opin Drug Metab Toxicol 11: 1665-1677.

4. Agnelli G, Gallus A, Goldhaber SZ, Haas S, Huisman MV, et al. (2007) Treatment of proximal deep-vein thrombosis with the oral direct factor $\mathrm{Xa}$ inhibitor rivaroxaban (BAY 59-7939): the ODIXa-DVT (Oral Direct Factor Xa Inhibitor BAY 59-7939 in Patients with Acute Symptomatic Deep-Vein Thrombosis) study. Circulation 116: 180-187.

5. Investigators E, Bauersachs R, Berkowitz SD, Brenner B, Buller HR, et al.
(2010) Oral rivaroxaban for symptomatic venous thromboembolism. N Engl J Med 363: 2499-2510.

6. Investigators E, Büller HR, Prins MH, Lensin AW, Decousus H, et al. (2012) Oral rivaroxaban for the treatment of symptomatic pulmonary embolism. N Engl J Med 366: 1287-1297.

7. Prins $M H$, Lensing $A W$, Bauersachs $R$, van Bellen $B$, Bounameaux $H$, et al. (2013) Oral rivaroxaban versus standard therapy for the treatment of symptomatic venous thromboembolism: a pooled analysis of the EINSTEINDVT and PE randomized studies. Thromb J 11: 21

8. Romualdi E, Donadini MP, Ageno W (2011) Oral rivaroxaban after symptomatic venous thromboembolism: the continued treatment study (EINSTEIN-extension study). Expert Rev Cardiovasc Ther 9: 841-844.

9. Wells PS, Prins MH, Levitan B, Beyer-Westendorf J, Brighton TA, et al. (2016) Long-term Anticoagulation With Rivaroxaban for Preventing Recurrent VTE: A Benefit-Risk Analysis of EINSTEIN-Extension. Chest 150: 1059-1068.

10. Ageno W, Mantovani LG, Haas S, Kreutz R, Monje D, et al. (2016) Safety and effectiveness of oral ricaroxaban versus standard anticoagulation for the treatment of symptomatic deep-vein thrombosis (Xalia): an international, prospective, non-interventional study. Lancet Haematol 3: e12-e21.

11. Keller L, Marten S, Hecker J, Werth S, Tittl L, et al. (2016) Treatment of acute VTE with rivaroxaban-Results of the prospective Dresden NOAC registry (NCT01588119). Blood 128: 2618.

12. Kucher N, Aujesky D, Beer JH, Mazzolai L, Baldi T, et al. (2016) Rivaroxaban for the treatment of venous thromboembolism. The SWIss Venous ThromboEmbolism Registry (SWIVTER). Thromb Haemost 116: 472-479.

13. Santos JT, Micco PD, Dentali F, Douketis J, Diaz-Peromingo JA, et al. (2017) Real-life treatment of venous thrombembolism with direct oral anticoagulants: The influence of recommended dosing and regimens. Thromb Haemost 117 382-389.

4. Moustafa F, Pierfranceschi MG, Micco PD, Bucherini $E$, Lorenzo A, et al. (2017) Clinical outcomes during anticoagulant therapy in fragile patients with venous thromboembolism. RPTH 1: 172-179.

15. Moustafa F, Pesavento R, Micco PD, Gonzalez-Martinez J, Quintavalla R, et al (2017) Real-life use of anticoagulants in venous thromboembolism with a focus on patients with exclusion criteria for direct oral anticoagulants. Clin Pharmacol Ther. 